der des $\mathrm{C}_{3} \mathrm{H}_{7} \mathrm{NH}_{2} / \mathrm{Fe}^{\oplus}$-Komplexes. Im MSMS-Experiment entstehen unimolekular die in Gleichung (b) aufgeführten Produkte, deren Genese durch Untersuchung der markierten Amine aufgeklärt wurde.

\begin{tabular}{|c|c|c|}
\hline & $\Delta m$ & $\begin{array}{l}\text { Neutral- } \\
\text { molekül }\end{array}$ \\
\hline$n \mathrm{C}_{3} \mathrm{H}_{7} \mathrm{NH}_{2} / \mathrm{Co}^{\oplus}$ & $\begin{array}{ll}\longrightarrow & 2 \\
& 28\end{array}$ & $\begin{array}{l}\mathrm{H}_{2} \\
\mathrm{C}_{2} \mathrm{H}_{4}\end{array}$ \\
\hline
\end{tabular}

Molekularer Wasserstoff stammt, wie auch bei $n \mathrm{C}_{3} \mathrm{H}_{7} \mathrm{NH}_{2} / \mathrm{Fe}^{\oplus}$, ausschließlich aus der $\beta$ - und $\gamma$-Position (1,2-Eliminierung); H-Gleichverteilung (Scrambling) wird nicht beobachtet.

Neutrales $\mathrm{CoH}$ entsteht durch $\mathrm{Co}^{\oplus}$-induzierte HydridAbstraktion ${ }^{[6.11]}$ aus der $\alpha$-Methylengruppe des Amins (Abspaltung von $\mathrm{CoD}$ aus $6 \mathrm{~b} / \mathrm{Co}^{\oplus}$ und von $\mathrm{CoH}$ aus den $\mathrm{Co}^{\oplus}$-Komplexen der übrigen Isotopomere von 6). Die Spezifität der Reaktion hängt vermutlich mit der leichten Bildung eines Iminium-Ions $\mathrm{CH}_{3} \mathrm{CH}_{2} \mathrm{CH}=\mathrm{N}^{\oplus} \mathrm{H}_{2}$ zusammen.

Im Gegensatz zu $\mathrm{Fe}^{\oplus}$ erzeugt $\mathrm{Co}^{\oplus}$ das Olefin aus der $\mathrm{C}(\boldsymbol{\beta}) / \mathrm{C}(\gamma)$-Position des Propylamins (Abspaltung von $\mathrm{C}_{2} \mathrm{H}_{4}$ statt $\mathrm{C}_{2} \mathrm{H}_{2} \mathrm{D}_{2}$ bei $6 \mathrm{~b} / \mathrm{Co}^{\oplus}$ ). Die Untersuchung der $\mathrm{Co}^{\oplus}$. Komplexe von $6 \mathrm{c}$ und $\mathbf{6 d}$ enthüllt allerdings, daß der Ethylen-Abspaltung ein unvollständiger Wasserstoffaustausch innerhalb der $\beta / \gamma$-Positionen vorgelagert sein muß. So entstehen aus $6 \mathrm{c} / \mathrm{Co}^{\oplus} \mathrm{C}_{2} \mathrm{H}_{2} \mathrm{D}_{2}$ und $\mathrm{C}_{2} \mathrm{H}_{3} \mathrm{D}$ im Verhältnis 2.8:1 und aus $6 \mathbf{d} / \mathrm{Co}^{\oplus} \mathrm{C}_{2} \mathrm{H}_{2} \mathrm{D}_{2}$ und $\mathrm{C}_{2} \mathrm{HD}_{3}$ im Verhältnis 2.7:1. Läge ein kompletter Austausch eines $\mathrm{A}_{3} \mathrm{~B}_{2}$-Systems vor $(A, B=H, D)$, so sollten aus $6 \mathrm{c} / \mathrm{Co}^{\oplus}$ und $6 \mathrm{~d} / \mathrm{Co}^{\oplus}$ die Isotopomere $C_{2} A_{2} B_{2}$ versus $C_{2} A_{3} B$ im Verhältnis 1.5:1 abgespalten werden. Vermutlich konkurriert die direkte OlefinEliminierung mit einer vorgelagerten Austauschreaktion vom Typ $11 \rightleftarrows 12$ (Schema 4). Unter Stoßaktivierung ent-

$\stackrel{(j)}{\mathrm{CH}_{3}}-\stackrel{(\beta)}{\mathrm{CH}_{2}}-\stackrel{(\alpha)}{\mathrm{CH}_{2}}-\mathrm{NH}_{2} / \mathrm{Co}^{\oplus} \longrightarrow$

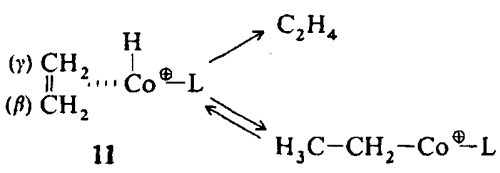

Schema 4. $\mathrm{L}=\mathrm{CH}_{2} \mathrm{NH}_{2}$.

12

steht ca. $20 \%$ Ethylen aus der $\mathrm{C}(\alpha) / \mathrm{C}(\beta)$-Position des Propylamins. Wahrscheinliche Zwischenstufe ist ein zu 8 (Schema 3) analoger $\mathrm{Co}^{\oplus}$-Komplex.

Eingegangen am 15. Dezember 1988 [Z 3088]

[1] a) J. Allison, D. P. Ridge, J. Am. Chem. Soc. 101 (1979) 4998; b) siehe auch S. J. Babinec, J. Allison, ibid. 106 (1984) 7718.

[2] a) C. B. Lebrilla, C. Schuize, H. Schwarz, J. Am. Chem. Soc. 109 (1987) 98 b) T. Drewello, K. Eckart, C. B. Lebrilla, H. Schwarz, Int. J. Mass Spectrom. Ion Processes 76 (1987) R 1; c) C. B. Lebrilla, T. Drewello, H. Schwarz, ibid. 79 (1987) 287; d) J. Am. Chem. Soc. 109 (1987) 5639; e) G. Czekay, T. Drewello, H. Schwarz, ibid., im Druck; f) Übersicht: G. Czekay, T. Drewello, K. Eller, C. B. Lebrilla, T. Prüsse, C. Schulze, N. Steinrück, D. Sülzle, T. Weiske, H. Schwarz in H. Werner, G. Erker (Hrsg.): Organometallics in Organic Synthesis, Springer, Berlin, im Druck.

[3] a) R. Breslow, Chem. Soc. Rev. 1 (1972) 553; b) Acc. Chem. Res. 13 (1980) 170 ; c) R. Breslow, A. Adams, T. Guv, J. Hunger, Lect. Heterocycl. Chem. 9 (1987) 43.

[4] ZAB-HF-3F-Massenspektrometer der Firma Vacuum Generators, BEBKonfiguration (B: magnetischer, E: elektrostatischer Sektor). Die in der
Ionenquelle erzeugten lonen wurden auf $8 \mathrm{keV}$ kinetische Energie beschleunigt, bei einem Auslösungsvermögen von ca. 3000 (10\% Tal-Definition) wurde $\mathrm{C}_{3} \mathrm{H}_{9} \mathrm{NFe}^{\oplus}$ mit BE selektiert; seine unimolekularen Zerfälle wurden durch "Scannen" von B(2) registriert. 5-50 Spektren wurden on-line mit dem VG-Datensystem 250/11 akkumuliett. Gerätebeschreibung siehe T. Weiske, Dissertation (D83), Technische Universität Berlin 1985.

[5] a) Wegen mangelnder Empfindlichkeit war es im vorliegenden Fall nicht möglich, die beim unimolekularen Zerfall der Komplexe entstehenden Neutralfragmente mit der ansonsten sehr nützlichen CIDI-Methode (Collisionally Induced Dissociative Ionization), einer Variante der Neutralisa tions-Reionisations-(NR-)Massenspektrometrie, zu charakterisieren; siehe b) P. C. Burgers, J. L. Holmes, A. A. Mommers, J. K. Terlouw, Chem. Phys. Lett. 102 (1983) 1; c) C. Wesdemiotis, F. W. McLafferty, Chem. Rev. 87 (1987) 485; d) J. K. Terlouw, H. Schwarz, Angew. Chem. 99 (1987) 829 Angew. Chem. Int. Ed. Engl. 26 (1987) 805; e) J. K. Terlouw, Adv. Mass. Spectrom. 11 (1989) 984

[6] Die durch $\mathrm{Co}^{\oplus}$ induzierte $\mathrm{HD}$-Abspaltung aus $\mathrm{CH}_{3} \mathrm{CH}_{2} \mathrm{ND}_{2}$ und die fehlende Wasserstoff-Abspaltung bei z. B. tert-Butylamin und Triethylamin veranlaßten Radecki und Allison zu der (unbegründeten) Verallgemeinerung, daß bei primären Aminen das Ưbergangsmetall-Ion vorwiegend die NH-Bindung aktiviert: B. D. Radecki, J. Allison, J. Am. Chem. Soc. 106 (1984) 946 (siehe auch Ergebnisse zum System Propylamin/Co ${ }^{\oplus}$ ).

[7] Definition: M. T. Reetz, Tetrahedron 29 (1973) 2189.

[8] Die $M^{\oplus}$-Olefin-Bindungsenergie beträgt für viele Systeme ca. 40$60 \mathrm{kcal} \mathrm{mol}^{-1}$; siehe z. B. M. A. Hanratty. J. L. Bea uchamp, A. J. Illies, P. van Koppen, M. T. Bowers, J. Am. Chem. Soc. 110 (1988) 1, zit. Lit. Die Barriere der Ethylen-Rotation in Komplexen des Typs (CO) ${ }_{4} \mathrm{Fe}$-Olefin is mit ca. 10-15 kcal mol ${ }^{-1}$ deutlich kleiner; siehe z. B. L. Kruczynski, L. K. K. Li Sing, Man J. Takats, J. Am. Chem. Soc. 96 (1974) 4006: S. T. Wilson, N. J. Coville, J. R. Shapley, J. A. Osborn, ibid. $96(1974) 4038 ;$ J. A. Segal. B. F. G. Johnson. J. Chem. Soc. Dalton Trans. 1975, 677, 1990.

[9] P. L. Watson, J. Am. Chem. Soc. 104 (1982) 337.

[10] a) T. Prüsse, C. B. Lebrilla, T. Drewello, H. Schwarz, J. Am. Chem. Soc. 110 (1988) 5986; b) K. Eller, T. Drewello, W. Zummack, T. Allspach, U. Annen, M. Regitz, H. Schwarz, ibid., im Druck; c) für ein „Gegenbeispiel“ aus der Chemie von Alkanen mit $\mathbf{M}^{\oplus}$ siehe R. Houriet, L. F. Haile, J. $L$. Beauchamp, Organometallics 2 (1983) 1818.

[11] S. W. Buckner, B. S. Freiser, J. Am. Chem. Soc. 109 (1987) 4715.

[12] Für bimolekulare Metathese-Reaktionen von Übergangsmetall-Ionen in der Gasphase siehe a) A. E. Stevens, J. L. Beauchamp. J. Am. Chem. Soc $101(1979) 6449$; b) L. F. Halle, P. B. Armentrout, J. L. Beauchamp, Orga nometallics $2(1983) 1829$; c) D. B. Jacobson, B. S. Freiser, J. Am. Chem Soc. 107 (1985) 2605; d) H. Kang, J. L. Beauchamp, ibid. 108 (1986) 5663 e) S. W. Buckner, J. R. Gord, B. S. Freiser, ibid. 110 (1988) 6606; I) S. W Buckner, B. S. Freiser, Polyhedron 7 (1988) 1583.

[13] a) J. L. Beauchamp, A. E. Stevens, R. R. Corderman, Pure Appl. Chem. 51 (1979) 967; b) J. Allison, Prog. Inorg. Chem. 34 (1986) 627.

\section{Photocycloadditionen des Benzvalens **}

\section{Von Manfred Christl* und Max Braun}

Schon 1975 berichteten Katz und Turro et al. ${ }^{[1]}$, daß bei der sensibilisierten Photolyse von Benzvalen 1 zwei Umlagerungen stattfinden können. Sensibilisatoren mit einer Triplettenergie $E_{\mathrm{T}}>65 \mathrm{kcal} \mathrm{mol}^{-1}$ bewirken die Umwandlung in Benzol, während solche mit $E_{\mathrm{T}}<63 \mathrm{kcal} \mathrm{mol}^{-1}$ die Automerisierung (1,3-C-Wanderung) auslösen. Als Sensibilisatoren waren hauptsächlich Ketone verwendet worden, die typische Substrate der Paterno-Büchi-Reaktion ${ }^{[2]}$ sind. Trotzdem erwähnten die Autoren keine Produkte mit Oxetan-Struktur. Angesichts der großen Aktivität des Benzvalens gegenüber Elektrophilen aller $\mathrm{Art}^{[3]}$ wollten wir wissen, ob nicht außer den genannten Isomerisierungen photochemische Cycloadditionen von 1 ablaufen.

Bei der Belichtung ${ }^{[4]}$ von $1^{[5]}$ in Gegenwart von Aceton und Benzophenon $\left(E_{\mathrm{r}}=78\right.$ bzw. $\left.69 \mathrm{kcal} \mathrm{mol}^{-1[2 \mathrm{a}]}\right)$ entstand Benzol, doch bildeten sich mit Biacetyl $\left(E_{\mathrm{T}}=\right.$ $\left.56 \mathrm{kcal} \mathrm{mol}^{-1[2 \mathrm{a}]}\right)$, Benzil (54 kcal mol $\left.{ }^{-1[2 \mathrm{a}]}\right)$, Phenylgly-

[*] Prof. Dr. M. Christl, Dipl.-Chem. M. Braun Institut für Organische Chemie der Universität Am Hubland, D-8700 Würzburg

[**] Die Arbeit wurde vom Fonds der Chemischen Industrie und von der Deutschen Forschungsgemeinschaft gefördert. 
oxylsäuremethylester $\left(61.9 \mathrm{kcal} \mathrm{mol}^{-1(6 a)}\right.$, Belichtung bei $\left.-30^{\circ} \mathrm{C}\right), 1,4-B e n z o c h i n o n\left(50 \mathrm{kcal} \mathrm{mol}^{-1[2 \mathrm{a}]}\right)$ und Chloranil $\left(56.4 \mathrm{kcal} \mathrm{mol}^{-1(6 \mathrm{~b}]}\right.$, Belichtung bei $\left.-30^{\circ} \mathrm{C}\right)$ die Oxetane $2(19 \%), 4(22 \%), 5(38 \%), 6(25 \%)$ bzw. $7(51 \%)^{[7]}$. Neben 2 beobachteten wir beim Versuch mit Biacetyl das Oxadihydrosemibullvalen $3(2: 3 \approx 30: 1)$, das auch zusammen mit einem geringen Anteil seines Diastereomers in $48 \%$ Ausbeute aus 2 durch Behandlung mit $\mathrm{AgBF}_{4}$ in $\mathrm{CDCl}_{3}$ bei $20^{\circ} \mathrm{C}$ erhältlich ist ${ }^{[7]}$. Der Versuch mit Chloranil lieferte nur bei $-30^{\circ} \mathrm{C}$ reines 7 ; bei $20^{\circ} \mathrm{C}$ entstand neben 7 das Umlagerungsprodukt $8^{[7]}(7: 8=4: 1)$. Ausgewählte physikalische Daten von 2-8 siehe Tabelle 1 .<smiles>CC(=O)C(C)=O</smiles>

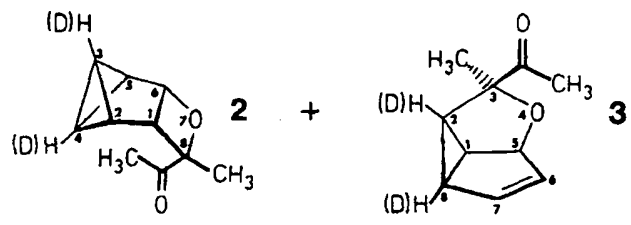

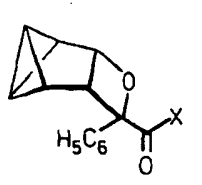

$4\left(x=\mathrm{C}_{6} \mathrm{H}_{5}\right)$ $5\left(X=\mathrm{OCH}_{3}\right)$

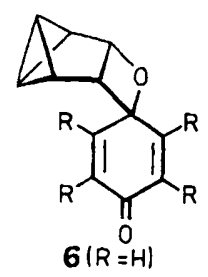

$7(R=C)$

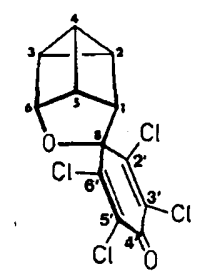

8
Die Bildung von 3 belegt den mehrstufigen Verlauf der Paterno-Büchi-Reaktion ${ }^{[2]}$. Aus Biacetyl im Triplettzustand und 1 geht wohl das Diradikal 9 hervor, das entweder zu 2 cyclisiert oder eine Cyclopropylmethyl-Homoallyl-Umlagerung $^{[3 a]}$ eingeht, die 10 und daraus 3 liefert, wenn in 9 von den beiden in Frage kommenden C-C-Bindungen die zum Biacetylteil cis-ständige Bindung bricht. In Übereinstimmung mit diesem Mechanismus resultierten beim Einsatz von $\left[1,6-\mathrm{D}_{2}\right]$ Benzvalen die dideuterierten Produkte [3,4$\left.\mathrm{D}_{2}\right] 2$ und $\left[2,8-\mathrm{D}_{2}\right] 3$.

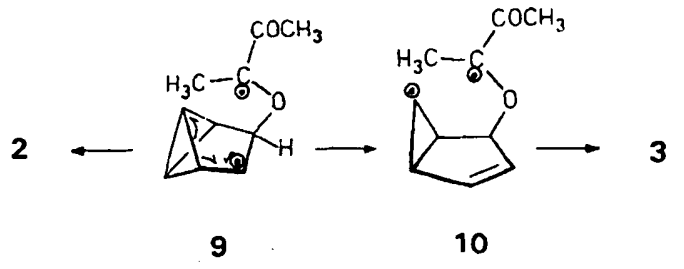

Der Befund, daß die Markierungen in 2 auf die Positionen 3 und 4 beschränkt sind ${ }^{[8]}$, schließt die Automerisierung von 1 durch den angeregten Sensibilisator Biacetyl aus. Zum gleichen Ergebnis kamen wir, indem wir unverbrauchtes $\left[\mathrm{D}_{2}\right] 1$ zurückgewannen und mit 2,4,6-Trimethylbenzonitriloxid umsetzten. Auch im resultierenden Dihydroisoxazol ${ }^{[9]}$ fanden sich die Deuteriumatome nur in den BicyclobutanBrückenkopf-Positionen ${ }^{[8]}$. Interessanterweise hatten Katz und Turro et al. ${ }^{[1]}$ Biacetyl mit in ihre Tabelle der verwendeten Sensibilisatoren aufgenommen, jedoch für die Automerisierung von 1 keine Quantenausbeute eingetragen. Letztere war bei Benzil mit ca. 0.2 angegeben worden. Wir bestätigten jetzt, da $\beta$ angeregtes Benzil die Automerisierung von 1 bewirkt. Aus $\left[1,6-D_{2}\right] 1$ ging $\left[D_{2}\right] 4$ hervor; das Markierungsverhältnis der Positionen 1(6), 2(5) und 3(4) betrug $1: 3: 45^{[8]}$. Laut Analyse des 2,4,6-TrimethylbenzonitriloxidAddukts waren im unverbrauchten $\left[D_{2}\right] 1$ die Markierungen stärker verteilt, nämlich im Verhältnis $1: 2: 4$ in den entsprechenden Positionen wie in $4^{[8]}$. Die Belichtung von $\left[1,6-D_{2}\right] 1$ in Gegenwart von 1,4-Benzochinon und 2-Benzoylthiophen (siehe unten) führte zu keiner 1,3-C-Wanderung in [1,6$\mathrm{D}_{2}$ ]1. Ob also Benzvalen 1 durch Ketone im Triplettzustand $\left(E_{\mathrm{T}}<63 \mathrm{kcal} \mathrm{mol}^{-1}\right)$ automerisiert wird, ist eine Frage relativer Reaktionsgeschwindigkeiten. Mit Biacetyl, 1,4-Benzochinon und 2-Benzoylthiophen läuft die Cycloaddition erheblich schneller $\mathrm{ab}$ als die für die Automerisierung von 1 notwendige Energieübertragung; bei Benzil sind die beiden Geschwindigkeiten ähnlich groß.

Die Struktur des Produkts 8 spricht für einen anderen Bildungsweg als den über ein Diradikal des Typs 9. Bekanntlich entsteht bei Belichtung von Chloranil in Gegenwart eines Donors ein Radikalionenpaar ${ }^{[10 a]}$, das im Falle von $\mathbf{1}$ als Donor durch CIDNP-Signale von 1 und Benzol nachgewiesen wurde ${ }^{[10 b]}$. Somit liegt nahe, daß 8 ein Folgeprodukt des Radikalionenpaars ist, das zum Zwitterion 11 weiterreagieren sollte. Nun müßte im kationischen Teil von 11 die Wagner-Meerwein-Umlagerung unter Wanderung des zum anionischen Teil trans-ständigen Bicyclobutan-Brükkenkopf-Kohlenstoffatoms ${ }^{[3]}$ ablaufen, woraus 12 und daraus 8 resultierte.

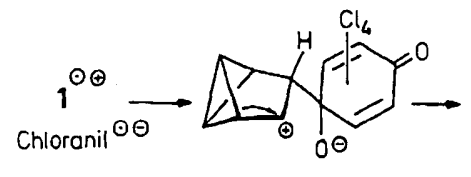

11

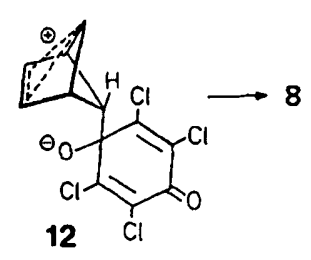

12
Anders als mit 1,4-Benzochinon ergab $1^{[5]}$ mit 1,4-Naphthochinon $\left(E_{\mathrm{T}}=57 \mathrm{kcal} \mathrm{mol}^{-1[6 \mathrm{c}]}\right)$ beim Belichten ${ }^{[4]}$ bei $-30^{\circ} \mathrm{C}$ das Cyclobutan $13^{[7]}(17 \%)$. Angeregt durch die Arbeiten von Cantrell ${ }^{[11]}$, setzten wir auch 2-Benzoylthiophen $\left(62.6 \mathrm{kcal} \mathrm{mol}^{-1(11)}\right)$ bei Raumtemperatur sowie 2Acetylthiophen $\left(64.5 \mathrm{kcal} \mathrm{mol}^{-1[11]}\right)$ und 2-Acetylfuran $\left(64.9 \mathrm{kcal} \mathrm{mol}^{-1(11)}\right)$ bei $-30^{\circ} \mathrm{C}$ ein und erhielten die [2 + 2]-Cycloaddukte $14(21 \%), 15(27 \%)$ bzw. das [4 + 2]Cycloaddukt $16(19 \%)$ und dessen Diastereomer $(6 \%)^{[7]}$. Aus angeregtem 1-Acetylnaphthalin $\left(56.4 \mathrm{kcal} \mathrm{mol}^{-1}{ }^{[6 \mathrm{a}]}\right)$ und $1^{[5]}$ entstand bei $-30^{\circ} \mathrm{C}$ zunächst das $[4+2]$-Cycloaddukt $17(25 \%)$, das jedoch mit ähnlicher Geschwindigkeit durch weiteres Belichten in $18(41 \%)$ und $19(9 \%)^{[7]}$ umge-

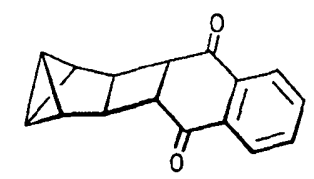

13

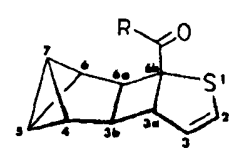

$14\left(R=\mathrm{C}_{6} \mathrm{H}_{5}\right)$ $15\left(\mathrm{R}=\mathrm{CH}_{3}\right)$

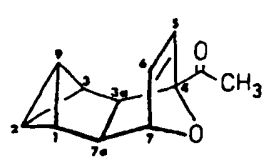

16

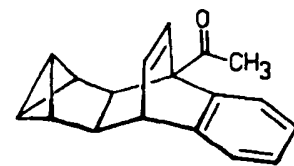

17

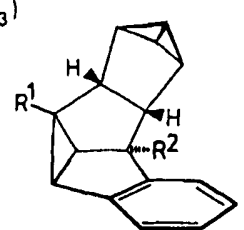

$18\left(R^{9}=\mathrm{COCH}_{3}, R^{2}=H\right)$ $19\left(R^{1}=\mathrm{H}, \mathrm{R}^{2}=\mathrm{COCH}_{3}\right)$ 
Tabelle 1. Ausgewähite physikalische Daten von 2-8 und 13-19; als Siedepunkt ist jeweils die Temperatur des Kugelrohrdestillationsapparats angegeben; die Zuordnung der Banden in den NMR-Spektren stützı sich auf NOE-Effekte und ${ }^{1} \mathrm{H}-{ }^{13} \mathrm{C}$-Korrelationen.

2: $\mathrm{Kp}=30-50: \mathrm{C} / 0.05$ Torr. $-\mathrm{IR}\left(\mathrm{CCl}_{4}\right): \tilde{v}=1713 \mathrm{~cm}^{-1}(\mathrm{C}=\mathrm{O}) .-{ }^{\mathrm{t}} \mathrm{H}-\mathrm{NMR}$ $\left(200 \mathrm{MHz}, \quad \mathrm{CDCl}_{3}\right): \delta=1.45 \quad\left(\mathrm{~s} ; \quad 8-\mathrm{CH}_{3}\right), 2.12 \quad(\mathrm{dtt}, \quad J(3,4)=8.8$, $J(2,4)=J(4,5)=1.8, \quad J(1,4)=J(4,6)=1.0 \mathrm{~Hz} ; 4-\mathrm{H}$ ), $2.17 \quad$ (br.dt. $J(2,3)=J(3,5)=1.7 \mathrm{~Hz} ; 3-\mathrm{H}), 2.25\left(\mathrm{~s} ; \mathrm{CO}-\mathrm{CH}_{3}\right), 2.31$ (br.dq, $J(2,5)=4.5$, $J(1.2)=1.7 \mathrm{~Hz} ; \quad 2-\mathrm{H}), \quad 2.54 \quad$ (dq. $J(5,6)=1.8 \mathrm{~Hz} ; 5-\mathrm{H}), 2.69$ (ddd, $J(1,6)=6.0 \mathrm{~Hz} ; 1-\mathrm{H}$ ), 4.71 (br.dd; 6-H). - ${ }^{13} \mathrm{C}-\mathrm{NMR}$ (50 MHz. $\mathrm{CDCl}_{3}$ ): $\delta=1.2(\mathrm{C}-4), 8.8(\mathrm{C}-3), 24.8$ und $25.2\left(2 \mathrm{CH}_{3}\right), 35.8(\mathrm{C}-2), 40.5(\mathrm{C}-5), 49.8$ (C-1), $80.1(\mathrm{C}-6), 87.2(\mathrm{C}-8), 213.6(\mathrm{C}=\mathrm{O})$

3: $\mathrm{Kp}=30-50^{\circ} \mathrm{C} / 0.05$ Torr. - IR $\left(\mathrm{CCl}_{4}\right): \tilde{v}=1710 \mathrm{~cm}^{-1}(\mathrm{C}=\mathrm{O})$. - 'H.NMR $\left(200 \mathrm{MHz}, \mathrm{CDCl}\right.$ ) $: \delta=1.40 \quad\left(\mathrm{~s} ; 3-\mathrm{CH}_{3}\right), 2.05$ (dddd, $J(2,8)=6.6$, $J(1,8)=6.0, J(7,8)=2.4, J(6,8)=0.6 \mathrm{~Hz} ; 8-\mathrm{H}), 2.17\left(\mathrm{~s} ; \mathrm{CO}-\mathrm{CH}_{3}\right), 2.27$ (ddd. $J(1,2)=7.7, J(2.6)=0.6 \mathrm{~Hz} ; 2-\mathrm{H}), 2.96(\mathrm{dddd}, J(1,5)=4.9, J(1,6)=0.5 \mathrm{~Hz}$; $1-\mathrm{H}), \quad 5.19 \quad($ ddd $, \quad J(5,6)=2.0, \quad J(5,7)=0.6 \mathrm{~Hz} ; 5-\mathrm{H}), \quad 5.46 \quad(\mathrm{ddq}$, $J(6,7)=5.4 \mathrm{~Hz} ; 6-\mathrm{H}), 5.84(\mathrm{ddd} ; 7-\mathrm{H}) .-{ }^{13} \mathrm{C}-\mathrm{NMR}\left(50 \mathrm{MHz}, \mathrm{CDCl}_{3}\right)$ : $\delta=25.8$ und $26.5\left(2 \mathrm{CH}_{3}\right), 31.7,36.6$ und 46.0 (C-1, C-2, C-8), 87.0 (C-5), 90.2 (C-3), 130.0 und $135.9(\mathrm{C}-6, \mathrm{C}-7), 215.1(\mathrm{C}=\mathrm{O})$

4: $\mathrm{Fp}=85-86^{\circ} \mathrm{C} .-\mathrm{IR}(\mathrm{KBr}): \tilde{v}=1670 \mathrm{~cm}^{-1}(\mathrm{C}=\mathrm{O})$

5: $\mathrm{Fp}=62-63^{\circ} \mathrm{C} .-\mathrm{IR}(\mathrm{KBr}): \hat{v}=1735 \mathrm{~cm}^{-1}(\mathrm{C}=\mathrm{O})$

6: $\mathrm{Fp}=72-73^{\circ} \mathrm{C} .-\mathrm{IR}(\mathrm{KBr}): \tilde{v}=1667,1661(\mathrm{C}=0), 1627,1602 \mathrm{~cm}^{-1}$ $(\mathrm{C}=\mathrm{C})$

7: $\mathrm{Fp}=140-142^{\circ} \mathrm{C} .-\mathrm{IR}(\mathrm{KBr}): \tilde{v}=1678(\mathrm{C}=\mathrm{O}), 1600.1568 \mathrm{~cm}^{-1}(\mathrm{C}=\mathrm{C})$ 8: $F p=163-165^{\circ} \mathrm{C} .-I R(\mathrm{KBr}): \tilde{v}=1680(\mathrm{C}=\mathrm{O}), 1607,1574 \mathrm{~cm}^{-1}(\mathrm{C}=\mathrm{C})$. - ${ }^{1} \mathrm{H}-\mathrm{NMR}\left(400 \mathrm{MHz}, \mathrm{C}_{6} \mathrm{D}_{6}\right): \delta=1.19$ (dddd, $J(2,5)=4.7, J(2,3)=4.5$, $J(2,4)=3.2, J(1,2)=2.6 \mathrm{~Hz} ; 2-\mathrm{H}), 1.70$ (ldd, $J(3,5)=4.5, J(3,4)=3.2$, $J(3,6)=2.2 \mathrm{~Hz} ; \quad 3-\mathrm{H}), \quad 1.98 \quad(\mathrm{td}, \quad J(4,5)=1.0 \mathrm{~Hz} ; 4-\mathrm{H}), 2.14 \quad(\mathrm{dd}$ $J(1,5)=4.5 \mathrm{~Hz} ; 1-\mathrm{H}), 2.60$ (quint. d, $J(5,6)=4.7 \mathrm{~Hz} ; 5-\mathrm{H}), 4.58(\mathrm{dd} ; 6-\mathrm{H})$. ${ }^{13} \mathrm{C}-\mathrm{NMR}\left(50 \mathrm{MHz}, \mathrm{CDCl}_{3}\right): \delta=15.5$ und $17.3(\mathrm{C}-2, \mathrm{C}-3), 22.3(\mathrm{C}-4), 48.6$ (C-5), 59.7 (C-1), 82.4 (C-6), 87.3 (C-8), 128.8 und $131.1\left(\mathrm{C}-3^{\prime}, \mathrm{C}-5^{\prime}\right), 153.4$ und $153.7\left(\mathrm{C}-2^{\prime}, \mathrm{C}^{-6^{\prime}}\right), 169.9\left(\mathrm{C}-4^{\prime}\right)$

13: $\mathrm{Fp}=155-156^{\circ} \mathrm{C} \cdot-\mathrm{IR}(\mathrm{KBr}): \tilde{v}=1665(\mathrm{C}=\mathrm{O}), 1592,1585 \mathrm{~cm}^{-1}(\mathrm{C}=\mathrm{C})$ 14: $F p=108-110^{\circ} \mathrm{C} .-I R(K B r): \tilde{v}=1679(C=O), 1594,1578 \mathrm{~cm}^{-1}(C=C)$. - ${ }^{1} \mathrm{H}-\mathrm{NMR} \quad\left(200 \mathrm{MHz}, \mathrm{CDCl}_{3}\right): \delta=1.86$ (br.s, 5-H, 7-H), 1.94 (dq, $J(4,6)=4.3, \quad J(6,6 \mathrm{a})=J(5,6)=J(6,7)=1.7 \mathrm{~Hz} ; \quad 6-\mathrm{H}), \quad 2.29 \quad(\mathrm{dq}$, $J(4,5)=J(3 \mathrm{~b}, 4)=J(4,7)=1.7 \mathrm{~Hz} ; \quad 4-\mathrm{H}), \quad 2.38 \quad(\mathrm{ddd}, \quad J(3 \mathrm{~b}, 6 \mathrm{a})=7.3$, $J(3 \mathrm{a}, 3 \mathrm{~b})=3.8 \mathrm{~Hz} ; 3 \mathrm{~b}-\mathrm{H}), 3.11$ (br.d; $6 \mathrm{a}-\mathrm{H}), 3.86(\approx \mathrm{tt}, J(3,3 \mathrm{a})=3.5$, $J(2,3 \mathrm{a}) \approx J(3 \mathrm{a}, 6 \mathrm{a}) \approx 1.1 \mathrm{~Hz} ; 3 \mathrm{a}-\mathrm{H}), 5.63(\mathrm{dd}, J(2,3)=5.9 \mathrm{~Hz} ; 3-\mathrm{H}), 6.09$ (dd; 2-H), $7.47(m-\mathrm{H}), 7.54(p-\mathrm{H}), 7.91(o-\mathrm{H}),-{ }^{13} \mathrm{C}-\mathrm{NMR}\left(50 \mathrm{MHz}, \mathrm{CDCl}_{3}\right)$ $\delta=0.9$ (C-7), 6.6 (C-5), 37.3 (C-6), 38.3 (C-4), 45.9 (C-3b), 52.7 (C-3a), 55.8 (C-6a), 63.0 (C-6b), $123.7(\mathrm{C}-3), 124.9(\mathrm{C}-2), 128.6(m-\mathrm{C}), 128.9(o-\mathrm{C}), 133.0$ (p-C), 135.1 (ipso-C), $195.3(\mathrm{C}=\mathrm{O})$

15: $\mathrm{Kp}=50-80^{\circ} \mathrm{C} / 0.01$ Torr. - IR (Film): $\tilde{v}=1705 \mathrm{~cm}^{-1}(\mathrm{C}=\mathrm{O})$

16: $\mathrm{Kp}=80^{\circ} \mathrm{C} / 0.01$ Torr. $-\mathrm{IR}\left(\mathrm{CCl}_{4}\right): \tilde{v}=1711 \mathrm{~cm}^{-1}(\mathrm{C}=\mathrm{O})$. ${ }^{1} \mathrm{H}-\mathrm{NMR}$ $\left(400 \mathrm{MHz}, \quad \mathrm{C}_{6} \mathrm{D}_{6}\right): \quad \delta=1.35 \quad(\mathrm{dtd}, \quad J(1,3)=4.5, \quad J(1,2)=J(1,9)=1.8$, $J(1.7 \mathrm{a})=1.0 \mathrm{~Hz} ; \quad 1-\mathrm{H}), \quad 1.63 \quad$ (cit, $\quad J(2,9)=8.3, \quad J(3,9)=1.8$, $J(3 \mathrm{a}, 9)=J(7 \mathrm{a}, 9)=1.2 \mathrm{~Hz} ; 9-\mathrm{H}), 1.68(\mathrm{dt}, J(2,3)=1.8 \mathrm{~Hz} ; 2-\mathrm{H}), 1.84(\approx \mathrm{dq}$, $J(3,3 \mathrm{a})=1.1 \mathrm{~Hz} ; 3-\mathrm{H}), 2.07\left(\mathrm{~s} ; \mathrm{CH}_{3}\right), 2.53(\mathrm{dt}, J(3 \mathrm{a}, 7 \mathrm{a})=8.1 \mathrm{~Hz} ; 3 \mathrm{a}-\mathrm{H}), 2.58$ (ddt, $J(7,7 \mathrm{a})=4.6 \mathrm{~Hz} ; 7 \mathrm{a}-\mathrm{H}), 4.39(\mathrm{dd}, J(6,7)=1.7 \mathrm{~Hz} ; 7-\mathrm{H}), 5.81$ (dd, $\left.J(5,6)=5.7 \mathrm{~Hz} ; 6-\mathrm{H}), 6.20(\mathrm{~d} ; 5-\mathrm{H}),-{ }^{13} \mathrm{C}-\mathrm{NMR}(50 \mathrm{MHz}, \mathrm{CDCl})_{3}\right): \delta=5.6$ und $9.3(\mathrm{C}-2, \mathrm{C}-9), 25.9\left(\mathrm{CH}_{3}\right), 33.3$ und $33.8(\mathrm{C}-1, \mathrm{C}-3), 49.8(\mathrm{C}-7 \mathrm{a}), 51.9$ (C-3a), 80.4 (C-7), $94.4(\mathrm{C}-4), 132.6$ und $134.2(\mathrm{C}-5, \mathrm{C}-6), 207.6(\mathrm{C}=\mathrm{O})$

17: $\mathrm{Kp}=140^{\circ} \mathrm{C} / 0.01$ Torr. $-\mathrm{IR}\left(\mathrm{CCl}_{4}\right): \tilde{v}=1711 \mathrm{~cm}^{-1}(\mathrm{C}=0)$

18: $\mathrm{Fp}=128-130^{\circ} \mathrm{C}$. - IR $(\mathrm{KBr}): \tilde{v}=1676 \mathrm{~cm}^{-1}(\mathrm{C}=\mathrm{O})$

19: Öl. - IR $\left(\mathrm{CCl}_{4}\right): \bar{v}=1708 \mathrm{~cm}^{-1}(\mathrm{C}=\mathrm{O})$

wandelt wurde (Di- $\pi$-Methan-Umlagerung). Ausgewählte physikalische Daten von 13-19 siehe Tabelle 1.

Kein Cycloaddukt erhielten wir aus 1 und Cyclopent-2-en1-on $\left(E_{\mathrm{T}}=74 \mathrm{kcal} \mathrm{mol}^{-1[2 \mathrm{a}]}\right)$. Ais Schlußfolgerung ergibt sich somit, daß auch 1 photochemische Cycloadditionen eingehen kann, vorausgesetzt, der Reaktionspartner hat eine Triplettenergie $E_{\mathrm{\tau}}<65 \mathrm{kcal} \mathrm{mol}^{-1}$.

Eingegangen am 7. Dezember $1988 \quad$ [Z 3078]

[1] C. A. Renner, T. J. Katz, J. Pouliquen, N. J. Turro, W. H. Waddell, J. Am. Chem. Soc. 97 (1975) 2568.

[2] a) N. J. Turro: Modern Molecular Photochemistry, Benjamin/Cummings, Menlo Park, 1978; b) W. M. Horspool (Hrsg.): Synthetic Organic Photochemistry, Plenum Press, New York 1984.

[3] a) M. Christl, Angew. Chem. 93 (1981) 515; Angew. Chem. Int. Ed. Engt. 20 (1981) 529, zit. Lit.; b) M. Christl, E. Brunn. F. Lanzendörfer, J. Am. Chem. Soc. 106 (1984) 373
[4] Lichtquellen: Hy-Mitteldrucklampe (Hanovia, $450 \mathrm{~W}$ ) im Pyrex-Tauchschacht, wobei im Falle der farbigen Substrate die Strahlung mit i $\leq 400 \mathrm{~nm}$ herausgefiltert wurde. Im Rayonet*-Reaktor RPR 100 wurden 1,4-Naphthochinon und 1-Acetylnaphthalin durch Bestrahlen mit 350-nm-bzw. 300-nm-Licht umgesetzt.

[5] Wir verwenden 1 als Lösung in Hexan, die mit der ein-bis fünffachen Menge Benzol (Versuche bei Raumtemperatur) oder Toluol (Versuche bei $-30^{\circ} \mathrm{C}$ ) verdünnt wurde. Die Lösungen, die vor der Photolyse mit Stickstoff gesättigt wurden, waren ca. $0.10 \mathrm{M}$ an 1 und $0.05 \mathrm{M}$ an Carbonylverbindung.

[6] a) W. G. Herkstroeter, A. A. Lamola, G. S. Hammond, J. Am. Chem. Soc, 86 (1964) 4537; b) P. Longin, A.-M. Lambert, M. A. Rousset, C.R. Acad. Sci. Ser. B 273 (1971) 599; c) G. S. Hammond, J. Saltiel, A. A. Lamola, N. J. Turro, J. S. Bradshaw, D. O. Cowan, R. C. Counsell, V. Vogt. C. Dalton, J. Am. Chem. Soc. 86 (1964) 3197.

[7] Die Isolierung gelang durch Blitzchromatographie mit Petrolether/Essigester an $\mathrm{SiO}_{2}(4-8,13-15)$ bzw. basischem $\mathrm{Al}_{2} \mathrm{O}_{3}$, Aktivitätsstufe III (1719), präparative Gaschromatographie an Carbowax 20M auf Volaspher* A $2(2,3)$ oder an Silicon SE 30 auf Chromosorb" W (16 und Diastereomer). Elementaranalysen, Massen-, IR- und NMR-Spektren stützen die vorgeschlagenen Strukturen. Außer bei 13 wurden die Konfigurationen aus den charakteristischen Größen der Kopplungskonstanten oder aus NOE-Effekten bestimmt.

[8] Analyse durch ${ }^{2} \mathrm{H}-\mathrm{NMR}$-Spektroskopie.

[9] M. Christl, B. Mattauch, H. Irngartinger, A. Goldmann, Chem. Ber. 119 (1986) 950.

[10] a) J. Mattay, Angew. Chem. 99 (1987) 849; Angew. Chem. Int. Ed. Engl. 26 (1987) 825 ; b) C. J. Abelt, H. D. Roth, M. L. M. Schilling, J. Am. Chem. Soc. 107 (1985) 4148.

[11] T. S. Cantrell, J. Org. Chem. 39 (1974) 2242.

\section{Hoch reduzierte Porphyrine **}

\section{Von Robert Cosmo, Christian Kautz, Klaus Meerholz,} Jürgen Heinze* und Klaus Müllen*

Kann ein organisches $\pi$-System durch sukzessive Aufnahme von Elektronen als Ladungsspeicher dienen, und wird die Speicherkapazität beim Übergang zu höhermolekularen Analoga mit mehreren Redoxzentren größer? Wir überprüfen dies erstmals für Porphyrine ${ }^{[1]}$ und weisen nach, daß der Zinkkomplex 1 des meso-Tetratolylporphyrins 2 reversibel sechs Elektronen aufnimmt. Die NMR-Spektren der intermediär auftretenden Di- und Tetraanionen belegen deren annulenähnliches Bindungsverhalten. Wir ,verdoppeln“" den Porphyrin-Zink-Komplex 1 zum stilbenverbrückten Diporphyrinylsystem 3 und untersuchen dessen Redoxverhalten in Analogie zu dem von 1. Der Komplex 3 bildet ein Dodecaanion!

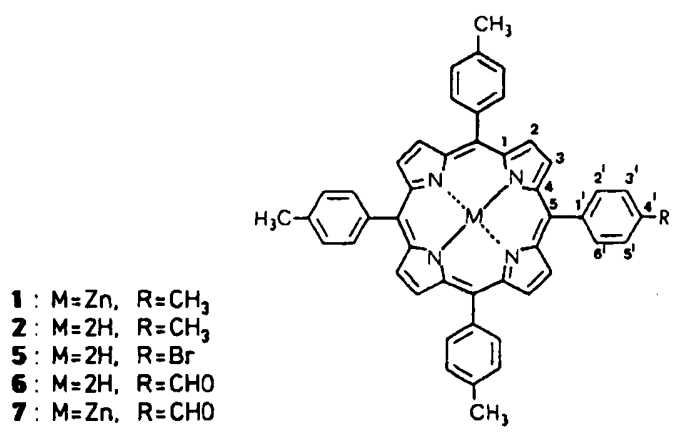

[*] Prof. Dr. K. Müllen, Dr. R. Cosmo, Dipl.-Chem. C. Kautz Institut für Organische Chemie der Universität J.-J.-Becher-Weg 18-22, D-6500 Mainz 1

Prof. Dr. J. Heinze, Dipl.-Chem. K. Meerholz Institut für Physikalische Chemie der Universität Albert-Straße 21, D-7800 Freiburg

[**] Diese Arbeit wurde von der Deutschen Forschungsgemeinschaft und dem Fonds der Chemischen Industrie gefördert. $R$. C. dankt der Alexandervon-Humboldt-Stiftung für ein Stipendium. 